Recepción: 21 / 06 / 2017

Aceptación: 25/ 07 / 2017

Publicación: 30 / 11 / 2017

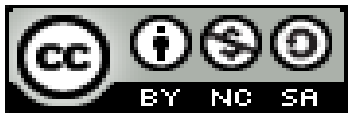

Ciencias de la educación

Artículo de investigación

\title{
Las técnicas grupales como instrumento andragógico para el aprendizaje teórico experimental en la formación pre-profesional
}

Group techniques as an andragogic instrument for experimental theoretical learning in preprofessional training

Las técnicas grupales como instrumento e para o aprendizado teórico experimental na formação pré-profissional

\author{
Ángel P. Acuña-Mejía ${ }^{\mathrm{I}}$ \\ dr_patricioacuna@yahoo.es \\ Fabio R. Bolaño-Valencia ${ }^{\text {II }}$ \\ fabiovalecia-12@gmail.com \\ Ana C. Bedoya-Gutiérrez III \\ ana-bedoya@hotmail.com \\ María G. Cedeño-Figueroa ${ }^{-1}$ V \\ kattycf_04hotmail.com
}

\section{Correspondencia:dr_patricioacuna@yahoo.es}

I Magister en Desarrollo del Talento Humano, Doctor en Ciencias Psicológicas, Docente en la Universidad Laica Eloy Alfaro de Manabí, Manta, Ecuador.

II Magister en Docencia Universitaria, Licenciado en Ciencias de la Educación Profesor de Enseñanza Secundaria en la Especialización de Física y Matemáticas, Doctor en Ciencias de la Educación Mención Enseñanza de la Matemática, Docente de la Universidad Técnica Luis Vargas Torres de Esmeraldas, Esmeraldas, Ecuador.

III Magister en Docencia Mención Gestión en Desarrollo del Currículo, Licenciada en Ciencias de la Educación Especialización Docencia Especial, Docente de la Universidad Técnica Luis Vargas Torres de Esmeraldas, Esmeraldas, Esmeraldas, Ecuador.

IV Profesora de Segunda Enseñanza en la Especialización de Asignaturas Secretariales, Licenciada en Ciencias de la Educación Especialidad Secretariado Bilingüe Computarizado, Unidad Educativa del Milenio "Carlos María Castro" Parroquia Santa Rita, Chone, Ecuador. 


\title{
Resumen
}

Se presenta una propuesta que lleva a considerar el uso de las técnicas grupales, como una herramienta andragógica para la enseñanza del estudiante universitario. Es el resultado de una larga experiencia en la investigación de campo. Se procede a asignar a los estudiantes en grupos de dos o más la tarea de investigar el tema asignado. Para cada subtema se encarga un estudiante, quien adquiere su posición personal del conocimiento adquirido. La sumatoria de los conocimientos adquiridos por parte de cada uno de los componentes del grupo permite crear posiciones personales, de cuya sumatoria se llega a un consenso en el grupo. Para esto se abren las opciones de las preguntas de los otros estudiantes de aula por cuyas participaciones se van construyendo criterios de conocimiento en los modelos mentales de cada estudiante.

Palabras clave: instrumento andragógico; formación pre-profesional; técnica grupal; aprendizaje.

\begin{abstract}
A proposal is presented that leads to consider the use of group techniques, as an andragogic tool for teaching university students. It is the result of long experience in field research. Students are assigned in groups of two or more the task of investigating the assigned topic. For each sub-topic a student is charged, who acquires his personal position of acquired knowledge. The sum of the knowledge acquired by each one of the members of the group allows the creation of personal positions, the sum of which reaches a consensus in the group. For this the options of the questions of the other students of classroom by whose participations are constructed criteria of knowledge in the mental models of each student open the options open.
\end{abstract}

Keywords: andragógico instrument; pre-vocational training; group technique; learning.

\section{Resumo}

Apresenta uma proposta que leva a considerar o uso de técnicas grupales, como uma ferramenta e pratica para a instrução do estudante universitário. É o resultado de uma larga experiência na investigação de campo. Se procede a asignar a los estudiantes em grupos de dos o mais a tarefa de investigar o assunto atribuído. Para cada subtema se encarga un estudiante, a quem pertence a posição pessoal do conhecimento adquirido. A sumatoria dos comprados adquiridos por parte de cada um dos componentes do grupo permite criar posições pessoais, de que sumatoria chegar a 
um consenso no grupo. Por favor, clique aqui para obter mais informações sobre os conceitos de conhecimento em modelos diferentes de cada estudante.

Palavras chave: instrumento andragógico; formação pré-profissional; técnica grupal; aprendizagem.

\section{Introducción}

La Segunda Guerra Mundial generó una fuerte inclinación por la conducta del agrupamiento, como una reacción ante la mirada altamente individual. En ese momento comienzan a cuestionarse ciertos problemas que iban apareciendo en la sociedad y que llegaron a su punto culminante con el avance del nazismo sobre todo en territorios geográficos y humanos. Se dio todo un contexto internacional que iba apoyando el pensamiento colectivo.

En Inglaterra, se destacaron W. R. Bion y S.H. Foulkes con la rehabilitación de soldados en el Hospital de Northfield; en Francia K. Lewin aplicó los principios gestálticos para el abordaje grupal y luego continuaron D. Anzieu, J.B. Pontalis y R Käes con los desarrollos psicoanalíticos. Respecto de J. Lacan, tuvo una actitud de rechazo hacia la cosa grupal, a la que consideraba 'como un reforzamiento obsceno del efecto imaginario del discurso, como estructura de alienación y desconocimiento’. (Cesio, 2004)

El trabajo en grupo consiste en "trabajar en coordinación con otros según acuerdos y metas establecidas para lograr un objetivo compartido" (Secretaría de Educación del Distrito, 2004).

Trabajar en equipo significa: "Un esfuerzo de concertación para llegar a metas comunes, formas de trabajo y mecanismos para regular el comportamiento. Trabajar en equipo no es estar reunidos en un espacio, en un mismo momento; es compartir ideales, formas de trabajo e intereses, es contar con un propósito común al que cada uno aporta”. (Barrios Jara, 1999).

Trabajar en equipo supone identificar lo que hace fuerte y débil al conjunto y tratar de hacer mejor el ritmo de trabajo de las personas que lo conforman.

El equipo de trabajo consiste en una agrupación de personas trabajando juntas, que comparten percepciones, tienen una propuesta en común, están de acuerdo con los procedimientos de 
trabajo, cooperan entre sí, aceptan un compromiso, resuelven sus desacuerdos en discusiones abiertas; lo anterior, no aparece automáticamente, sino que debe irse construyendo poco a poco. Estamos hablando de una acción colaborativa donde la discusión no es el objetivo sino el medio. (Barrios Jara, 1999).

Camilloni (2012), se refiere al trabajo en grupo como una modalidad de enseñanza que promueve el aprendizaje activo, centrado en el estudiante y que le brinda múltiples oportunidades de confrontar sus ideas con los otros miembros del grupo. El estudiante evalúa, acepta e intercambia argumentos y perspectivas, exponiendo también sus ideas a juicio del resto del equipo. La evaluación del trabajo en equipo no se limitará al resultado final, sino que estará presente en todo el transcurso del proceso de elaboración del proyecto. Convendrá evaluar grupalmente la comprensión y cumplimiento de la consigna, planificación del proyecto, participación de los integrantes y la capacidad de recibir feedback y autoevaluarse.

"La evaluación individual, en cambio, requerirá criterios específicos a cada miembro del grupo, como la asistencia a las reuniones, la cantidad y calidad de aportes al proyecto, el tiempo dedicado, la capacidad de motivar a los demás, de reconocer los errores, y la buena disposición a corregirlos. El cumplimiento del rol que toma cada integrante y la coordinación y dirección del grupo, son factores de evaluación muy importantes para el proyecto grupal" (Camilloni, 2012).

"En el Siglo XXI la educación ha reconocido en el estudiante a un sujeto activo en dicho proceso y al docente como un provocador de éste. El éxito para la enseñanza estriba en los procesos que median entre la enseñanza y el aprendizaje, éstos son procesos de carácter cognitivo que se desarrollan en el interior del alumno. Las Técnicas para el Proceso de enseñanza-aprendizaje con modelos andragógicos, en la mediación pedagógica en la educación superior en este caso son un puente conector, que facilitan la comunicación, la interacción y la transposición del conocimiento del docente a un conocimiento didáctico que pueda ser comprendido por el estudiante." (Jimenez Moreno, 2016).

La Andragogía "es la disciplina que se ocupa de la educación y el aprendizaje del adulto. Andragogía es al adulto, como Pedagogía al Niño. El adulto busca el conocimiento para su aplicación inmediata que le permita redituar en el menor tiempo, existiendo la clara conciencia de 
buscar ser más competitivo en la actividad que el individuo realice, más aún si el proceso de aprendizaje es patrocinado por una organización que espera mejorar su posición competitiva, mejorando sus competencias laborales, entendiendo como competencias al conjunto de conocimientos, habilidades, destrezas y actitudes orientadas a un desempeño superior en su entorno laboral, que incluyen tareas, actividades y responsabilidades, que contribuyen al logro de los objetivos clave buscados." (Yturralde, s/f).

A lo largo de la historia, la andragogía ha sido conceptualizada como:

- Una ciencia (Félix Adam, 1970).

- La ciencia de la educación de los adultos (Ludojoski, 1971).

- Un conjunto de supuestos (S. Brookfield, 1984).

- Un método (Lindeman, 1984).

- Una serie de lineamientos (Merriman, 1993).

- Una filosofía (Pratt, 1993).

- Una disciplina (Brandt, 1998).

- Una teoría (Knowles, 2001).

- Como proceso de desarrollo integral del ser humano (Marrero, 2004).

- Un modelo educativo (Universidad Nacional Experimental Simón Rodríguez, 1999)." (Caraballo Colmenares, 2007).

Ideas fuerza de la andragogía:

- "Idea fuerza 1. El reconocimiento de que los estudiantes o agentes de aprendizaje son sujetos adultos con la capacidad de asumir responsabilidades en todos los ámbitos de la vida; por ello en los procesos educativos andragógicos, la actividad se centra en el aprendizaje del sujeto siendo él quien tiene que asumir la responsabilidad de sus procesos de aprendizaje. Aprende debido a que quiere aprender para transformar su situación personal y social.

- Idea fuerza 2. Los intereses y necesidades del adulto deben ser compatibles con las demandas económicas, políticas y culturales de la sociedad. La andragogía 
responsabiliza al adulto de sus aprendizajes, en un proceso de formación a lo largo de la vida, esto se relaciona estrechamente con el empleo de los avances tecnológicos y la consecuente mejora del nivel económico y social.

- Idea fuerza 3. La confrontación permanente entre teoría y práctica y el análisis del proceso constituye un aspecto importante del proceso andragógico, al obligar a los docentes o andragogos y estudiantes o agentes de aprendizaje, a desarrollar su creatividad crítica e innovadora.

- Idea fuerza 4. Las instituciones educativas que diseñan y desarrollan procesos andragógicos, tienen la responsabilidad de crear y recrear valores hacia el trabajo socialmente útil. Por ello debe existir una vinculación estrecha entre los procesos de trabajo y los hechos andragógicos" (Pérez, 2009).

El objetivo de este trabajo es presentar una propuesta que lleva a considerar el uso de las técnicas grupales como una herramienta andragógica para la enseñanza del estudiante universitario.

\section{Metodología}

Para la presentación de la propuesta se hace un análisis documental de las fuentes de información, relacionadas con las técnicas grupales y la andragogía.

Se realiza la propuesta a partir de una experiencia. La temática de una técnica grupal es siempre elaborada mediante una técnica investigativa, en la que el profesor está consciente del trabajo a realizar, por lo que el motivo de la investigación tendrá la suficiente base cognitiva por parte del catedrático. Se pueden indicar algunas técnicas grupales tales como: seminario, mesa redonda, debate o controversia, simposio, discusión en grupos pequeños, técnicas de la reñida, desempeño de roles, plenario, panel, entre otros.

\section{Desarrollo}

Lo primero que se hace es explicar a los estudiantes qué se va hacer y cómo se va a realizar el estudio, para que lograr una conciencia en la labor a realizar. "Esta concepción educativa debe fundamentarse en una filosofía que reconozca como significativa la toma de conciencia de la 
problemática particular que enfrentan los adultos para obtener una profunda y comprometida participación en sus procesos de aprendizaje personales y colectivos.” (Pérez, 2009).

Se procede a asignar a grupos de dos o más estudiantes la tarea de investigar un tema asignado. "Para que el aprendizaje sea significativo es necesario proporcionar problemas interesantes, pertinentes y atractivos de resolver. Además, los alumnos deben estar activos, tienen que manipular algo (elaborar un producto, manipular parámetros, tomar decisiones) e influir de alguna forma en el entorno.” (García Irles, de la Sen Fernández, Marco de la Calle, Vázquez Araújo, \& Sempere Ortells, s/f).

De cada subtema se encarga un estudiante, quien adquiere su posición personal del conocimiento adquirido pero que transmite a los demás del grupo llegando a un consenso en equipo. Es decir, se toma en cuenta a Maturana (1996), al facilitar la convivencia, las relaciones interpersonales y la reflexión que posibilita que surjan conocimientos nuevos. Se favorece un entorno de responsabilidad ética, desde la reflexión y el respeto por el individuo. Se crean espacios donde se materializan las orientaciones dadas por el profesor y cuyo resultado no depende de la obediencia, ni de la rigidez de su realización, sino de la co-inspiración participativa en un proyecto común. "Todo conocimiento es un modo de vivir, por lo tanto, es multidimensional y su multidimensionalidad debe adquirirse en el vivir.

De la misma forma, que una de las dimensiones de existencia de los seres humanos es su relación con los otros como ser humano, con un modo particular de ser en un dominio relacional en el conversar, su coordinación con el hacer y el emocionar, que lo conlleva a construir realidades. El mundo que cada uno de nosotros vive siempre se configura con otros, a través de relaciones interpersonales, y surge en la dinámica de nuestro operar.” (Maturana, 1996).

Además, se abren las opciones de las preguntas de los otros estudiantes de aula por cuyas participaciones se van construyendo criterios de conocimiento en los modelos mentales de cada estudiante.

Esto favorece la ayuda, el compartir, el colaborar y el cooperar que "se expresan como norma permanente, propiciada en el quehacer de los proyectos como una interdependencia positiva, que 
cambia la competitividad por la construcción de buenas relaciones entre los diferentes miembros del equipo, permitiendo acceder a nuevos espacios de desarrollo cognitivo individual y grupal." (Trujillo Varga, 1998).

Es necesario destacar que la confrontación entre enfoques y puntos de vista, permitirá al auditorio obtener una información variada sobre el asunto que se esté tratando. En la utilización profesional de estas técnicas grupales en varias de ellas existe un director moderador, que es quien cumple un papel muy importante ya que es quien controla el tiempo de exposición, arbitra situaciones comportamentales, dirige órdenes de exposiciones, preside, coordina, enlaza el grupo y es la persona que tiene el suficiente conocimiento del tema a ser tratado, en conclusión, viene a ser el árbitro del evento.

Sobre las personas que debaten se debe condicionar emocionalmente a estos y para que acusen mayor empeño en el trabajo investigativo a ser expuesto o defendido en que ellos son unos expertos en el tema, (palabra estratégica pronunciada en momento y hora oportunas) que motiva al expositor a empeñarse y tratar de lucir en la presentación de sus resultados, además de recordarle que entra en una cancha donde habrá contendientes que competirán sobre el mismo tema. Dentro de los componentes de una técnica grupal, está el público que no viene a ser un simple observador, sino que debe considerarse como parte de la misma contienda ya que son personas que conocen del tema a ser tratado.

En la actividad andragógica desarrollada se pueden reconocer con cierta facilidad las condiciones que las caracterizan:

Confrontación de experiencias entre adultos: "La riqueza del ser adulto radica en su experiencia, en lo que sabe, lo que ha vivido, lo que ha realizado, lo que piensa y siente. La experiencia humana es heterogénea, y enriquecer esa experiencia para mejorar, adaptarse y poder resolver de manera satisfactoria las problemáticas a las que nos enfrentemos, es una de las principales motivaciones para diseñar e implementar procesos educativos permanentes. En la actividad andragógica, se desvanece la diferencia entre educador y educando. Ambos sujetos son adultos, con experiencias por su participación social. El concepto tradicional de uno que enseña y otro que aprende, uno que ignora y otro que sabe, en teoría deja de existir en la actividad andragógica para 
convertirse en una acción recíproca. La actividad andragógica es un proceso dinámico, activo, real, concreto, objetivo y esencialmente práctico. El adulto asiste a una institución escolar o inicia de manera individual un proceso de formación para enriquecer su cultura, para actualizar o acrecentar los conocimientos que ya posee, dinamizar su experiencia, mejorar profesionalmente o simplemente satisfacer intereses y necesidades que se estructuran en su vida cotidiana." (Pérez, 2009).

El adulto posee elementos de juicio que le permiten reflexionar sobre los contenidos educativos. Racionalidad que le conduce a ampliar sus motivaciones para el logro de sus intencionalidades educativas.

La capacidad de abstracción del adulto: "El adulto sabe perfectamente por qué y para qué participa en un proceso educativo, por lo tanto, puede apreciar con cierta sencillez y de manera ya sea deductiva o inductiva las consecuencias de sus actos educativos. Es importante subrayar que la actividad educativa de los adultos se funda en la voluntad y no en la imposición. El adulto estudia, aprende, se forma en torno a finalidades que el mismo ha establecido y que en muchas ocasiones ignora el propio educador. A diferencia de los niños y jóvenes, el adulto promueve su educación, la planea y la lleva a cabo en función de sus propias necesidades e intereses inmediatos y con miras a mejorar y consolidar un futuro mejor.” (Pérez, 2009).

El estudiante integra a su vida y aplica en su medio social las nuevas experiencias: "Proceso de integración y aplicación que tiene un carácter funcional que asegura, acrecienta y diversifica las motivaciones y vivencias del adulto. Cuando este integra a su vida cotidiana sus nuevos aprendizajes, genera un esfuerzo competitivo en su entorno social. Entran en juego las capacidades de los diferentes adultos que se relacionan en ese espacio a fin de imponer su liderazgo y demostrar su suficiencia. Esta acción competitiva, propia de toda acción humana debe ser considerada en la actividad andragógica. La integración de las viejas experiencias con las nuevas y su aplicación al trabajo, así como a la vida social, deben orientarse de tal manera que el sujeto adulto se esfuerce cotidianamente para contribuir a su bienestar y al de la sociedad a la que pertenece.” (Pérez, 2009). 


\section{Conclusiones}

Con la técnica empleada, los estudiantes:

- Adquirieron mayores conocimientos basados en sus investigaciones,

- hicieron mayor demostración de conocimientos adquiridos,

- hubo una mayor seguridad en el contenido de sus presentaciones con pérdida del miedo escénico,

- se logró mayor interés en conocer y demostrar las actividades que conllevan un proceso de investigación científica.

\section{Referencias Bibliográficas}

Barrios Jara, N. (1999). El aula un escenario para trabajar en equipo. Recuperado el febrero de 2016, de www.gestiopolis.com

Camilloni, A. (2012). La evaluación de trabajos elaborados en grupo. En A. Camilloni, La Evaluación Significativa. Buenos Aires: Paidós

Caraballo Colmenares, R. (2007). La andragogía en la educación superior. Investigación y postgrado, Caracas, Universidad Pedagógica Experimental Libertador, vol. 22, núm. 2, 187-206

Cesio, S. (2004). Teoría y técnica de grupos. Revista internacional de psicología 5 (1)

García Irles, M., de la Sen Fernández, M., Marco de la Calle, F., Vázquez Araújo, B., \& Sempere Ortells, J. (s/f). La construcción del conocimiento en el proceso enseñanza-aprendizaje: una herramienta de evaluación formativa

Jimenez Moreno, L. (2016). Técnicas para el proceso enseñanza-aprendizaje con modelos andragógicos en la mediación pedagógica en la educación superior. Séptima conferencia mundial de educación

Maturana, H. (1996). El sentido de lo humano. Dolmen ediciones 
Pérez, U. (2009). Modelo andragógico. Fundamentos. México: Universidad del Valle de México

Secretaría de educación del distrito. (2004)

Trujillo Varga, J. (1998). Trabajo en equipo, una propuesta para los procesos de enseñanza aprendizaje. IV Congresso RIBIE. Brasilia

Yturralde, E. (s/f). Andragogía es al adulto, como pedagogía al niño. Recuperado el febrero de 2016, de http://www.talleresdeandragogia.com/ 